\title{
Oculopharyngeal involvement in familial neurogenic muscular atrophy
}

\author{
M. MATSUNAGA ${ }^{1}$, T. INOKUCHI, A. OHNISHI, AND Y. KUROIWA \\ From the Department of Neurology, Neurological Institute, Faculty of Medicine, \\ Kyushu University, Fukuoka, Japan
}

SUMMARY A Japanese family with progressive spinal muscular atrophy is presented. Seven members in two generations were affected and the mode of inheritance was probably an autosomal recessive trait. A characteristic feature of this family was the presence of oculopharyngeal involvement in some of the affected members, in addition to the variable distribution of muscular atrophy among each of the affected members, such as mainly proximal or distal atrophies in the limbs. In one case the oculopharyngeal weakness appeared without limb involvement. The changes in the extremities were thought to be of neurogenic origin, and so was the progressive external ophthalmoplegia seen characteristically in these cases, although the latter was similar to ocular myopathy.

Involvement of the external ocular muscles is considered rare in progressive spinal muscular atrophy and in its variants (Merritt, 1967), although the facial and bulbar muscles are sometimes affected in such neurogenic diseases (Magee, 1960; Tsukagoshi, Nakanishi, Kondo, and Tsubaki, 1965; Araki and Sugimoto, 1966; Fenichel, Emery, and Hunt, 1967; Kennedy, Alter, and Sung, 1968; Tsukagoshi, Shoji, and Furukawa, 1970).

On the other hand, chronic progressive external ophthalmoplegia had been thought of uniformly as myopathy since the report of Kiloh and Nevin (1951). Their opinion, however, was criticized recently both clinically (Daroff, Solitare, Pincus, and Glaser, 1966) and experimentally (Drachman, Wetzel, Wasserman, and Naito, 1969).

This paper concerns a Japanese family in which progressive spinal muscular atrophy with a great inter-individual variation of muscular involvement occurred in seven family members within two generations. Two of the four cases examined had mainly distal muscle atrophy in the limbs with oculopharyngeal involvement; one case showed only oculopharyngeal involve-

1 Present address: The Third Department of Internal Medicine, Hirosaki University School of Medicine, Hirosaki, Japan. ment similar to oculopharyngeal myopathy while the other had proximal muscular atroph ${ }_{0}$ without oculopharyngeal involvement. There was evidence that the limb muscle atrophies as well as the oculopharyngeal were manifestations of the same disease, which was of primarily neurogenic origin. The case of oculopharyngea involvement without weakness in the extremities is of special value in discussing the pathogenesis of progressive external ophthalmoplegia.

\section{CASE 1}

$(I V-12)$ A 61 year old man complaining of blepharoptosis, nasal voice, and weakness in the extremities was admitted on 24 April 1970 to the Department of Neurology, Kyushu University Hospital. He had been in good health until the onset of nasal voice in the latter half of his fifth decade. A few years later, gait disturbance appeared insidiously and he began to stumble easily. These symptoms evolved progressively for the first few years but became apparently stationary thereafter. The age of onset of blepharoptosis was not clear because the patient did not notice it in its early stages, but photographs allowed us to conclude that this sign most probably appeared when the patient was about the age of 50 years. From a year before admission he observed slight dysphagia and double vision on upper and lateral gaze. 


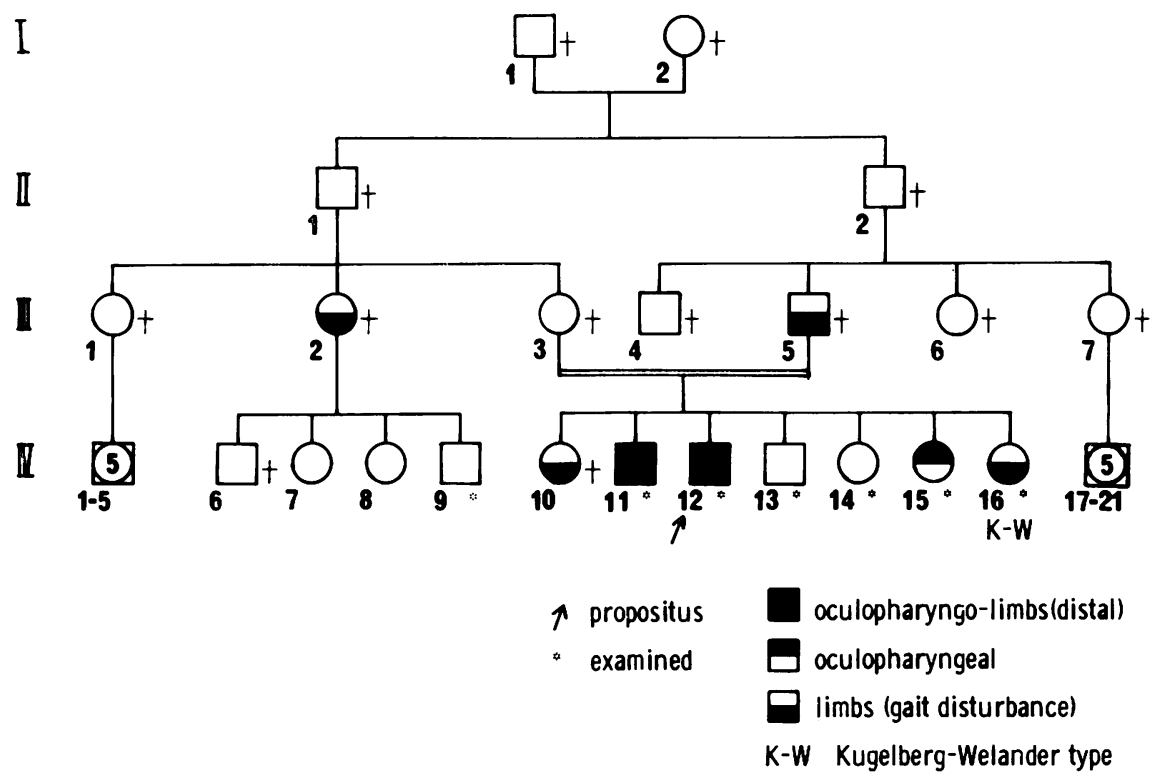

FIG. 1. Family tree.

FAMILY HISTORY A similar disorder was observed in the patient's father, an aunt, and several siblings described in detail below (Fig. 1).

NEUROLOGICAL EXAMINATION The patient was a small, emaciated man. There was no mental disturbance. The optic discs and retinae were normal.
Ocular movements were impaired markedly in the vertical and moderately in the horizontal directions. There was double vision on upper and lateral gaze. Pupillary light response and convergence reflex were present but sluggish. Marked bilateral ptosis with compensatory wrinkling of the forehead and slight lagophthalmos were observed (Fig. 2). The muscles

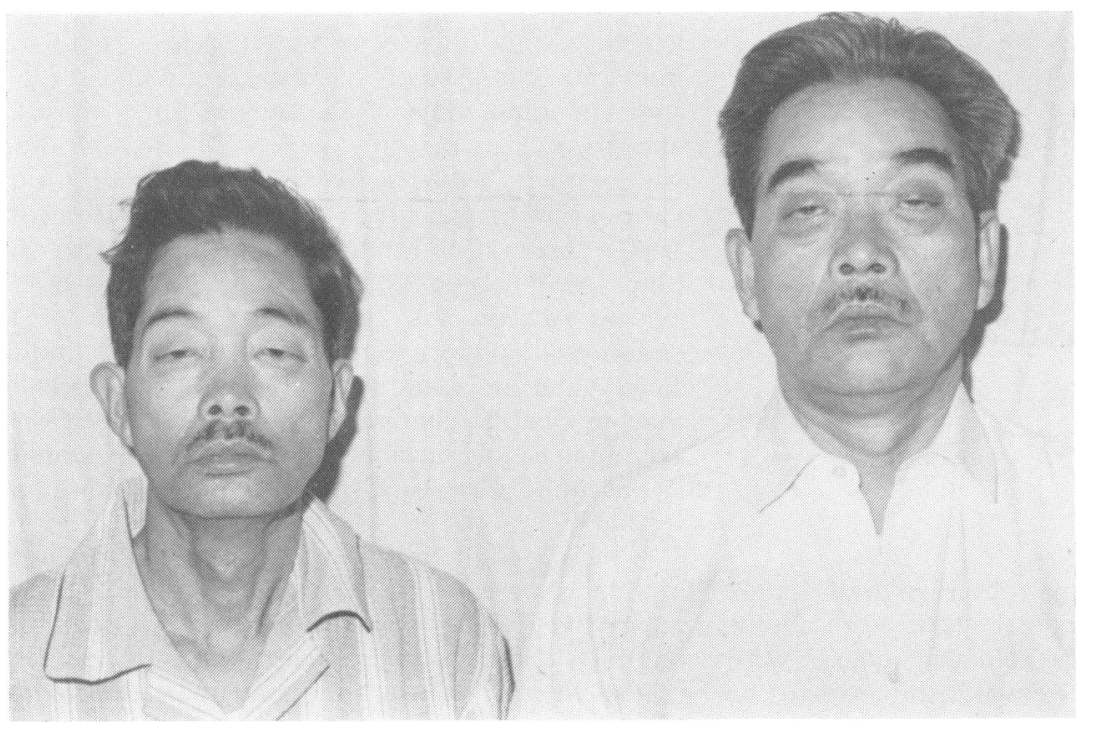

FIG. 2. Case $1(I V-12)$ at left and case $4(I V-I I)$ at right. 
of facial expression were moderately involved and he was unable to keep his eyes shut during forced opening of the eyes. His speech was markedly dysarthric and movement of the soft palate was poor, but the tongue was not atrophic and neck flexion was moderately weak. The proximal muscles of all limbs were slightly weak and distal portions were severely affected, especially the small muscles of the hands and the ankle flexor groups (Fig. 3). He had steppage gait and was not able to walk on the toes or heels.

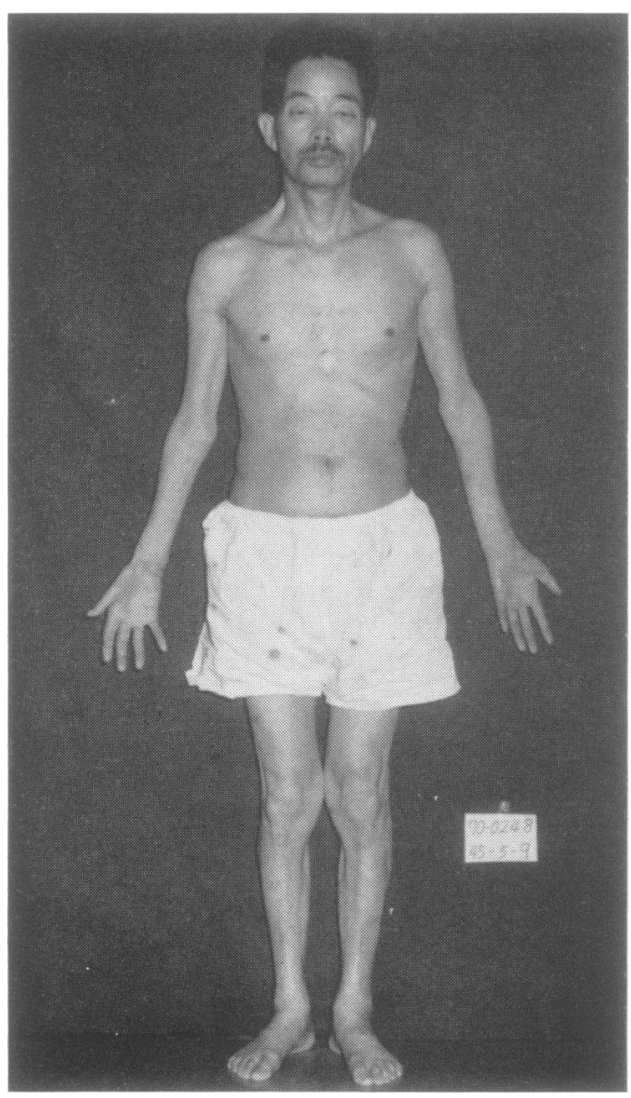

FIG. 3. Case 1. Note the marked muscular atrophy, especially in the distal portions of the limbs.

Fasciculations were sometimes observed in the hands and upper thighs. Deep tendon reflexes were absent in all extremities and no pathological reflexes were observed. There was no sensory impairment or ataxia.
LABORATORY EXAMINATIONS Routine examinations including the complete blood count, erythrocyte sedimentation rate, serum electrolytes, urinalysis, cerebrospinal fluid, electrocardiogram, and radiographic examinations of the skull and spine were all normal. Serum creatin phosphokinase activity was elevated to $59 \cdot 3$ units (normal: 0 to 19). Urinary excretion of 17-ketosteroids, 17-hydroxycorticosteroids, creatinine, and creatine were within normal limits.

ELECTROMYOGRAPHIC STUdies (Dr. Y. Murai and Dr. K. Mukuno) Electromyography (EMG) of the right biceps, first dorsal interosseous, thenar, quadriceps femoris, and anterior tibial muscles showed a reduced number of motor unit action potentials on maximum voluntary contraction and 'giant' motor unit potentials of long duration. The right thenar muscle revealed fibrillation potentials and 'giant' action potentials exceeding $6 \mathrm{mV}$. These data support a neurogenic origin of the muscle atrophy. Electromyography of the right sternocleidomastoid and orbicularis oris muscles was normal. Ocular EMG of the right orbicularis oculi, left lateral $\stackrel{\mathbb{D}}{\circ}$ rectus, and left medial rectus muscles showed a웅 tendency toward low voltage potentials $(50-200 \mu \mathrm{V}) \stackrel{\varnothing}{\varnothing}-$ on maximum contraction but a normal number of $\mathbb{D}$ action potentials was observed.

Motor nerve conduction velocities of the righto ulnar and tibial nerves were normal (ulnar 50.4 $\mathrm{m} / \mathrm{sec}$, tibial $40.7 \mathrm{~m} / \mathrm{sec}$ ).

MUSCLE BIOPSIES Specimens were taken from the left soleus and gastrocnemius muscles. There were moderate changes of the muscle architecture. In some bundles, most of the muscle fibres were disappearing, leaving only a few small-sized fibres, while others were relatively well spared, though various-sized small fibres were seen among normalsized fibres. Central emigration of nuclei was observed in some fibres although it was not so evident. There was considerable increase of the endomysial connective tissue and fat. These findings suggested a longstanding denervation pattern rather than myopathic changes (Fig. 4). On histochemical examination of succinate dehydrogenase staining, it was impossible to distinguish type I fibres from type II.

A biopsy of the right levator palpebrae superioris muscle showed a few muscle fibres buried in markedly increased connective tissue. The atrophy of the muscle was so severe that it could not be determined whether it was of myogenic or neurogenic origin (Fig. 5). 


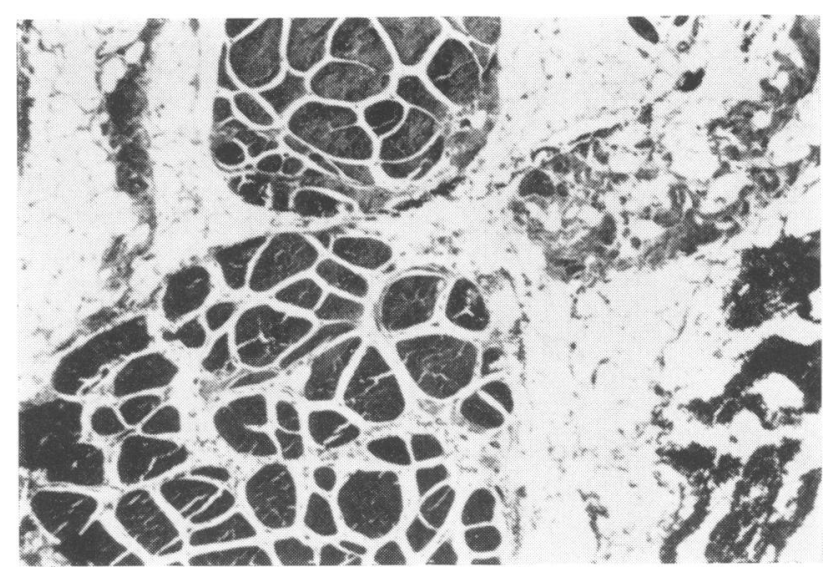

FIG 4. Biopsy specimen from the left gastrocnemius muscle of case 1, cut in cross section. In some of the bundles, most of the muscle fibres are disappearing leaving a few small-sized fibres, while the others are relatively well spared, though varioussized small firbes are seen among normalsized fibres. Endomysial connective tissue and fat are increased. $H$ and $E, \times 60$.

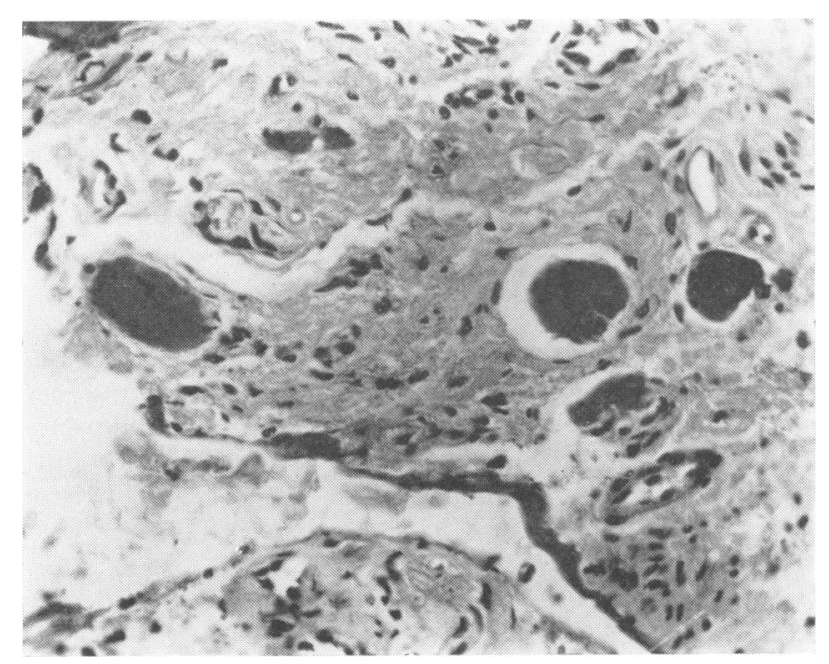

FIG. 5. Biopsy specimen from the right levator palpebrae superioris muscle of case 1, cut in cross section. The endomysial connective tissue is increased and a few muscle fibres spared are of small calibre. $H$ and $E, \times 270$.

CASE 2

(IV-16) A 50 year old woman, the youngest sister of case 1, was admitted on 12 June 1970. She had been in good health and an active sportswoman until a gait disturbance of insidious onset appeared when she was 35 years old. At that time her disability was apparently intermittent. Within a few years, however, her gait disturbance became continuous and slowly progressive. From a few years before admission she even needed to be assisted by the arms in climbing stairs or rising from a squatting position. After the age of 45 years, she began to feel difficulty holding her arms above the head. Recently she had occasionally noticed fasciculations in the shoulders and upper thighs.

NEUROLOGICAL EXAMINATION Examination revealed an alert, intelligent woman with no acute distress. Muscles innervated by the cranial nerves were intact, except for a slight weakness of the left trapezius muscle (Fig. 6). The shoulder girdle muscles and proximal muscles of the arms were affected moderately. Weakness was prominent in the left deltoid and triceps muscles. The distal muscles of the upper limbs were of normal strength and not atrophic. The iliopsoas and upper thigh muscles were moderately weak and Gowers' sign was present. The gastro- 


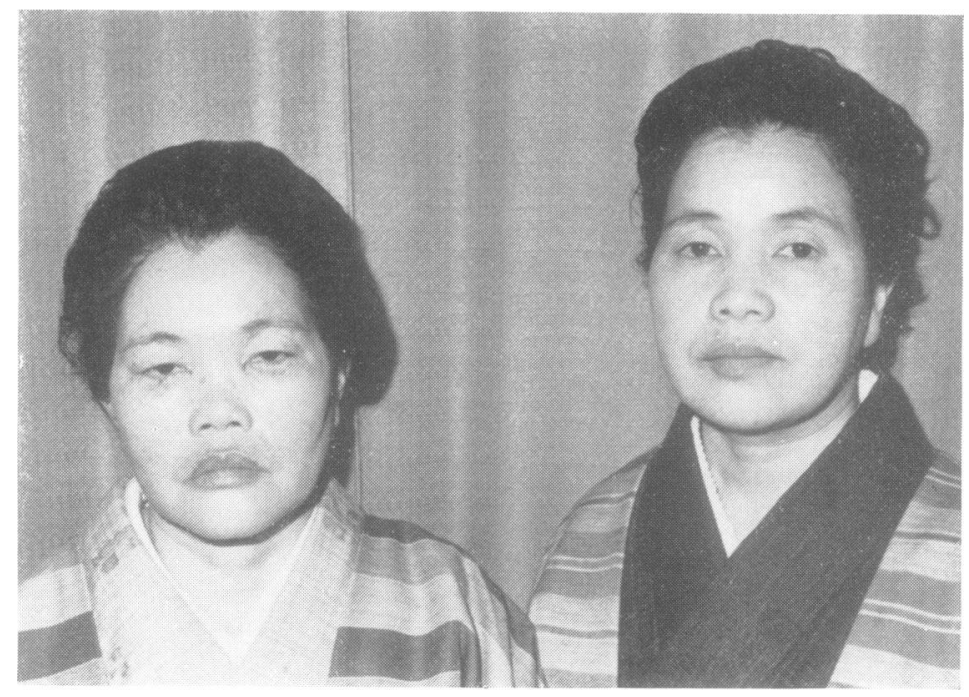

FIG. 6. Case $3(I V-15)$ at left and case $2(I V-16)$ at right. Case 3 showed moderate extraocular and facial muscle involvement without weakness in the extremities, while, in case 2, muscles innervated by cranial nerves were intact in spite of proximal muscle atrophy in the limbs.

cnemius and anterior tibial muscles were slightly weak. Her gait was waddling. Deep tendon reflexes were all absent and no pathological reflexes were observed. No sensory impairment and no ataxia were observed.

LABORATORY DATA The complete blood count, erythrocyte sedimentation rate, serum electrolytes, and protein fractions, liver function, urinalysis, radiography of the spine and chest, and electrocardiogram were all normal. Urinary excretion of 17-ketosteroids, 17-hydroxycorticosteroids, and creatinine were normal, but creatine excretion was slightly increased. Serum creatin phosphokinase activity was $75 \cdot 8$ units.

ELECTROMYOGRAPHIC STUdies (Dr. Y. Murai) EMG was performed on the right sternocleidomastoid, deltoid, biceps, first dorsal interosseous, and quadriceps femoris muscles. The motor unit potentials on maximum voluntary contraction were diminished, and 'giant' potentials of 5 to $8 \mathrm{mV}$ with a total duration of 10 to $20 \mathrm{msec}$ were recorded in all these muscles except the sternocleidomastoid. The interference patterns were incomplete, especially in the deltoid and quadriceps femoris muscles. These findings were interpreted as being compatible with a neurogenic atrophy.

The motor nerve conduction velocities of the left ulnar and tibial nerves were normal (ulnar 55.9 $\mathrm{m} / \mathrm{sec}$, tibial $44.2 \mathrm{~m} / \mathrm{sec}$ ).

MUSCLE BIOPSY The left vastus medialis muscle was chosen for the biopsy as weakness was of moderate degree. Small sized angulated fibres of 10 to $20 \mu$ in diameter were seen in groups or disseminately, butổ no alteration in gross architecture was observed. Sarcolemmal nuclei were often increased withoutī central emigration.

\section{CASE 3}

$(I V-I 5)$ A 55 year old woman, a sister of cases 1 and 2 , began suffering from hoarseness in the fourth decade (exact age of onset uncertain) followed by slight dysphagia and ptosis when she was about 45 years old. All these symptoms followed a slowly progressive evolution. She had never felt weakness of the extremities before this.

When examined in May 1970 she was alert and there was no mental disturbance. The pupils were of normal size, isocoric, and reacted promptly to light. Upward and horizontal eye movements were moderately restricted on the right and slightly on the left. Depression of the eyes was not impaired. There was moderate bilateral ptosis (Fig. 6). The orbicularis oculi muscles were weak bilaterally. She was dysarthric and movement of the soft palate was somewhat limited. The cranial nerves showed no other abnormalities. In the extremities, even when inspection revealed a slight emaciation of the distal portions, muscle power was normal. All deep tendon reflexes were absent and no pathological reflexes were observed. There was no sensory impairment or ataxia.

EMG of the right orbicularis oris, deltoid, and first dorsal interosseous muscles showed no pathological findings. That of the right inferior rectus 
muscle showed a tendency to low voltage (30 to $100 \mu \mathrm{V})$ without any changes which suggested denervation. On the other hand, action potentials recorded from the levator palpebrae superioris muscle were more than $500 \mu \mathrm{V}$ or sometimes polyphasic exceeding $6 \mathrm{msec}$, and the number of action potentials was reduced on maximum contraction.

Serum creatin phosphokinase activity was 10.3 units.

\section{CASE 4}

(IV-1I) A 63 year old man, the eldest brother in the family, noticed bilateral ptosis from about the age of 50 years. His condition progressed gradually and dysarthria and dysphagia continued from several years before the examination. Otherwise he felt no disability.

On examination, pupillary responses were normal and the ocular fundi were also normal. There was marked bilateral ptosis compensated by a posterior head tilt (Fig. 2). Horizontal eye movements were slightly restricted though he did not complain of diplopia. Elevation and depression of the eyes were normal. The facial muscles were slightly atrophic. There was a slight degree of nasal voice and also a slight dysphagia. The tongue was not atrophic. Strength was slightly weak in the distal muscles of the limbs and atrophy of the small muscles of the hands was observed. All tendon reflexes were absent. Tandem gait, gait on toes and heels and monopedal jumping were all normal.

Creatin phosphokinase activity was elevated to 76.6 units.

He refused EMG examination.

OTHER MEMBERS IN PEDIGREE III-2 Female. After the age of 45 years, weakness of both legs occurred. This followed a slowly progressive course until her death at the age of 72 years. Involvement of the upper limbs was uncertain.

III-3 Mother of cases 1-4. She was reported to have been normal until her death of cerebral apoplexy at the age of 61 years.

III-5 Father of cases 1-4. He suffered from a gait disturbance from the age of 45 years. The symptom was slowly progressive throughout all his later life. It was uncertain whether the muscles in the upper limbs were affected or not.

$I V-6$ and $I V-8$ They presented no neurological signs but methaemoglobinaemia had been diagnosed by their home doctor.

IV-10 She presented a slight degree of gait disturbance which appeared a few years before her death of pulmonary tuberculosis at the age of 37 years.

$I V-13$ and $I V-14$ They were examined by the authors, confirming the absence of any neurological abnormality. Serum creatin phosphokinase activities were also within normal limits.

Generation $V$ Among the members of the fifth generation, 47 members, several as old as 40 years of age, presented apparently no neurological disorders.

\section{DISCUSSION}

A characteristic feature of this family was the presence of limb muscle atrophy, which could be of distal or proximal type, associated with oculopharyngeal involvement in some of the affected members (Table). In one case oculopharyngeal involvement occurred in the absence of limb

TABLE

AFFECTED MEMBERS IN FAMILY

\begin{tabular}{|c|c|c|c|c|c|c|c|c|c|}
\hline \multirow[t]{2}{*}{ Case } & \multirow[t]{2}{*}{ Sex } & \multirow{2}{*}{$\begin{array}{c}\text { Age of } \\
\text { onset } \\
(y r)\end{array}$} & \multirow[t]{2}{*}{ Initial symptoms } & \multicolumn{5}{|c|}{ Muscles involved } & \multirow{2}{*}{$\begin{array}{l}C P K^{*} \\
\text { (units) }\end{array}$} \\
\hline & & & & ocular & facial & $\begin{array}{c}\text { pharyn- } \\
\text { geal }\end{array}$ & upper limbs & lower limbs & \\
\hline 11I-2 & $\mathrm{F}$ & $45 ?$ & Gait disturbance & - & - & - & $?$ & + & \\
\hline III-5 & $\mathbf{M}$ & 45 & Gait disturbance & - & - & - & ? & + & \\
\hline IV-10 & $\mathrm{F}$ & 35 & Gait disturbance & - & - & - & $\dot{-}$ & + & \\
\hline IV-11 (case 4) & $\mathbf{M}$ & 50 & Blepharoptosis & + & + & + & $+($ distal $)$ & \pm (distal) & $76 \cdot 6$ \\
\hline IV-12 (case 1) & $\mathbf{M}$ & 45 & Nasal voice & + & + & + & + (dist. > prox.) & $+($ dist. $>$ prox. $)$ & $59 \cdot 3$ \\
\hline IV-15 (case 3) & $\mathbf{F}$ & 35 & Hoarseness & + & + & + & - & - & $10 \cdot 3$ \\
\hline IV-16 (case 2) & $\mathbf{F}$ & 35 & Gait disturbance & - & - & - & $+($ proximal $)$ & $+($ proximal $)$ & $75 \cdot 8$ \\
\hline
\end{tabular}

+ Affected, \pm questionable, - spared.

* Creatin phosphokinase activity (normal, 0 to 19 units). 
atrophy. Although this case could not be differentiated clinically from oculopharyngeal myopathy, electromyographic studies and biopsies of the limb muscles of the other members in the family verified that the condition was of neurogenic origin. We considered our patients as variant cases of progressive spinal muscular atrophy. Neither a slight elevation of serum creatin phosphokinase activity nor the presence of central emigration of nuclei and increase of endomysial fibrous tissue in biopsy studies can be considered contradictory to the neurogenic origin of the muscle atrophy in our cases, since such findings are often observed in neurogenic diseases such as Kugelberg-Welander disease (Kondo, 1967), Charcot-Marie-Tooth disease (Lucas and Forster, 1962), longstanding anterior poliomyelitis (Drachman, Murphy, Nigam, and Hills, 1967), and neurogenic scapuloperoneal muscular atrophy (Kaeser, 1966; Haase and Shy, 1960).

As we had occasion to follow up only two generations in which the disease appeared clinically, and since the consanguineous marriage made the pedigree complicated, it was very difficult to decide the mode of inheritance. An autosomal recessive trait was most probable, but an autosomal dominant trait could not be ruled out completely at this stage. Besides, considering that we were dealing with rare diseases, the possibility that the two rare conditions of progressive external ophthalmoplegia and spinal muscular atrophy run independently in the family was highly improbable. We therefore considered both to have originated from a single genetic abnormality. Even if, in general, a constant genotype may present different phenotypes in hereditary diseases, the great variability of the phenotypes observed in this family was determined partially by the late onset of the disease and the presence of the consanguineous marriage.

As described in the introduction section, bulbar and facial muscles are sometimes involved in progressive spinal muscular atrophy and its variants, and even several cases with blepharoptosis have been reported before. Progressive external ophthalmoplegias, however, are thought to be exceptional in progressive spinal muscular atrophy. Nevertheless this possibility was suggested previously by a few authors: Myrianthopoulos and Brown (1954) reported two families in which some cases of progressive external ophthalmoplegia were seen. Kaeser (1965) reported about 12 affected members in a family with scapuloperoneal muscular atrophy. In some of them the facial and pharyngeal muscles were also involved, and in one case even external ophthalmoplegia occurred. Aberfeld and Namba (1969) reported a case with Kugelberg-Welander disease with progressive external ophthalmoplegia. There is also evidence that ocular muscle involvement of neurogenic origin may present a clinical picture which mimics ocular myopathy. Daroff et al. (1966) described a case of central nervous degenerative disease of unknown aetiology which had started clinically with progressive external ophthalmoplegia mimicking ocular myopathy. A levator palpebrae muscle biopsy in the early stage of their case led them to confirm the diagnosis of ocular myopathy. Nevertheless, necropsy revealed that it was a case of spongiform encephalopathy with special involvement of the brain-stem including supra-o nuclear, nuclear, and internuclear structures con- $-\mathbb{D}$ cerned with eye movements. They concluded唡 that the myogenic origin of ocular muscle atrophy cannot be determined by muscle biopsy alone, criticizing the tendency, after the reporto of Kiloh and Nevin (1951), to suspect a myogenic origin in almost all cases of progressive external ophthalmoplegia based primarily on extraocular muscle biopsy or electromyographic studies. These clinical observations were supported by Drachman et al. (1969) experimentally in dogs. They made intracranial lesions by avulsing or crushing the oculomotor nerve. After killing the animals in the period from two weeks to one year, they proved that histological changes of the extraocular muscles showed a picture characteristic of a primary myopathy including inflammatory, degenerative, and regenerative processes.

This particular difficulty in histological evaluation of the external eye muscles is most probably related to the fact that one single motor unit innervates an extremely small number of muscle fibres in these muscles. For the same reason, the EMG findings in ocular muscles in the differential diagnosis are still a matter of discussion. For example, Teasdall and Sears (1960) reported electromyographic findings in six cases with clinically diagnosed ocular myopathy. In three of 
them rather neuropathic changes such as a reduced number of action potentials or polyphasic motor unit potentials were recorded. As in case $3(\mathrm{IV}-15)$ of the present family, the electromyographic studies of the right inferior rectus muscle showed typical patterns frequently seen in ocular myopathy. On the other hand, those of the levator palpebrae superioris muscle were suggestive of denervation changes. Therefore, we could not determine whether the external ophthalmoplegia in our cases was of neurogenic or of myogenic origin directly from the electromyographic findings of extraocular muscles. The absence of a clear neurogenic pattern of biopsy and electromyographic studies of the extraocular muscles in our cases would in no instance exclude the possibility of a neurogenic origin of the condition.

These facts lead us to conclude that the oculopharyngeal involvement seen in our cases was of neurogenic origin, and should be considered as a partial symptom of progressive spinal muscular atrophy.

The authors are grateful to Dr. Y. Murai in our department who performed the electrophysiological studies on the limbs, and to Dr. K. Mukuno, Department of Ophthalmology, Kyushu University, for his coordination of ocular EMG and the muscle biopsy of the extraocular muscles.

\section{REFERENCES}

Aberfeld, D. C., and Namba, T. (1969). Progressive ophthalmoplegia in Kugelberg-Welander disease: report of a case. Archives of Neurology, 20, 253-256.

Araki, S., and Sugimoto, N. (1966). A pedigree of hereditary progressive bulbar-spinal muscular atrophy. Clinical Neurology (Tokyo), 6, 13-19.

Daroff, R. B., Solitare, G. B., Pincus, J. H., and Glaser, G. H.
(1966). Spongiform encephalopathy with chronic progressive external ophthalmoplegia: central ophthalmoplegia mimicking ocular myopathy. Neurology (Minneapolis), 16, 161-169.

Drachman, D. B., Murphy, S. R., Nigam, M. P., and Hills J. R. (1967). 'Myopathic' changes in chronically denervated muscle. Archives of Neurology, 16, 14-24.

Drachman, D. A., Wetzel, N., Wasserman, M., and Naito, H. (1969). Experimental denervation of ocular muscles: a critique of the concept of 'ocular myopathy'. Archives of Neurology, 21, 170-183.

Fenichel, G. M., Emery, E. S., and Hunt, P. (1967). Neurogenic atrophy simulating facioscapulohumeral dystrophy: dominant form. Archives of Neurology, 17, 257-260.

Haase, G. R., and Shy, G. M. (1960). Pathological changes in muscle biopsies from patients with peroneal muscular atrophy. Brain, 83, 631-637.

Kaeser, H. E. (1965). Scapuloperoneal muscular atrophy. Brain, 88, 407-418.

Kennedy, W. R., Alter, M., and Sung, J. H. (1968). Progressive proximal spinal and bulbar muscular atrophy of late onset: a sex-linked rccessive trait. Neurology (Minneapolis), 18, 671-680.

Kiloh, L. G., and Nevin, S. (1951). Progressive dystrophy of the external ocular muscles (ocular myopathy). Brain, 74, $115-143$.

Kondo, K. (1967). The muscle biopsy findings and the electromyographic changes in Kugelberg-Welander's disease. Brain and Nerve (Tokyo), 19, 133-139.

Lucas, G. J., and Forster, F. M. (1962). Charcot-MarieTooth disease with associated myopathy: a report of a family. Neurology (Minneapolis), 12, 629-636.

Magee, K. R. (1960). Familial progressive bulbar-spinal muscular atrophy. Neurology (Minneapolis), 10, 295-305.

Merritt, H. H. (1967). A Textbook of Neurology, 4th edn. Lea and Febiger: Philadelphia.

Myrianthopoulos, N. C., and Brown, I. A. (1954). A genetic study of progressive spinal muscular atrophy. American Journal of Human Genetics, 6, 387-411.

Teasdall, R. D., and Sears, M. L. (1960). Ocular myopathy: clinical and electromyographic considerations. Archives of Neurology, 2, 281-292.

Tsukagoshi, H., Nakanishi, T., Kondo, K., and Tsubaki, T. (1965). Hereditary proximal neurogenic muscular atrophy in adult. Archives of Neurology (Chicago), 12, 597-603.

Tsukagoshi, H., Shoji, H., and Furukawa, T. (1970). Proximal neurogenic muscular atrophy in adolescence and adulthood with X-linked recessive inheritance: KugelbergWelander disease and its variant of late onset in one pedigree. Neurology (Minneapolis), 20, 1188-1193. 